\title{
CRITICAL BEHAVIOUR OF WEAKLY DISORDERED SYSTEMS WITH REPLICA SYMMETRY BREAKING POTENTIALS
}

\author{
P. V. Prudnikov, V. V. Prudnikov \\ Department of Theoretical Physics, Omsk State University \\ $55 a$, Pr. Mira, Omsk, 644077, Russia
}

(Received August 30, 2001; received in final form September 24, 2001)

\begin{abstract}
A field-theoretic description of the critical behaviour of the weakly disordered systems is given. Directly, for three- and two-dimensional systems a renormalization analysis of the effective Hamiltonian of the model with replica symmetry breaking (RSB) potentials is carried out in the two-loop approximation. For the case with first-step RSB the fixed points (FPs) corresponding to the stability of the various types of critical behaviour are identified with the use of the Padé-Borel summation technique. The analysis of FPs has shown a stability of the critical behaviour of the weakly disordered three- and two-dimensional systems with respect to RSB effects and realization of the former scenario of disorder influence on critical behaviour. For the case of systems with arbitrary dimension from 3 to 4 the ranges of critical behaviour determined by the RSB effects are found for each value of the $p$-component order parameter without the use of $\varepsilon$-expansion. A comparison with the results of the $\varepsilon$-expansion calculations is carried out.

Key words: renormalization group, disordered systems, replica symmetry breaking potentials, field-theoretic approach, Padé-Borel summation technique.

PACS number(s): 64.60.Ak, 64.60.Fr, 64.60.Ht
\end{abstract}

The effects produced by weak quenched disorder on the critical phenomena have been studied for many years [1-5]. According to the Harris criterion [1], the disorder affects the critical behaviour only if $\alpha$, the specific heat exponent of the pure system, is positive. In this case a new universal critical behaviour, with new critical exponents, was established. In contrast, when $\alpha<0$, the disorder appears to be irrelevant for the critical behaviour.

In dealing with the weak quenched disorder the traditional approach is the replica method [4,5], and in terms of replicas all the results obtained for the disorder systems correspond to the so-called replica-symmetric (RS) solutions. Physically this means that only a unique ground state is assumed to be relevant for the observable thermodynamics. However, in a number of papers [6-8] ideas about replica symmetry breaking (RSB) in the systems with quenched disorder were presented. For the first time in [6] physical arguments showing that in the presence of the quenched disorder there exist numerous local minimal-energy configurations separated by finite barriers and a demonstration of how the summation over these local minimum configurations can provide additional RSB interaction potentials for fluctuating fields were offered.

The renormalization group (RG) description of the classical $\phi^{4}$ model with RSB potentials was presented in the one-loop approximation using $\varepsilon$-expansion [6-8]. It was shown that the RSB degrees of freedom produce a dramatic effect on the asymptotic behaviour of the RG flows, such that for a general type of RSB there exist no stable fixed points (FPs), and RG equations arrive in the strong-coupling regime. In contrast, in [9] using the fermion representation it was demonstrated that the crit- ical behaviour of the $2 \mathrm{D}$ weakly disordered Ising system is stable with respect to the RSB modes.

However, our numerous investigations of pure and disordered systems performed in the two-loop and higher orders of the approximation for the $3 \mathrm{D}$ system directly together with methods of series summation show that the predictions made in the lowest order of the approximation, especially on the basis of the $\varepsilon$-expansion, can differ strongly from the real critical behaviour [10]. Therefore, the results of RSB effects investigation in [6-8] must be reconsidered with the use of a more accurate fieldtheoretic approach in the higher orders of the approximation.

In this paper, we realize the field-theoretical RG description in the two-loop approximation of the $3 \mathrm{D}$ and $2 \mathrm{D}$ models and model with arbitrary dimension from three to four of the weakly disordered systems with RSB interaction potentials of forth order on fluctuating fields. We carry out the solution of the RG equations with the use of the series summation method and the analysis of stability of various types of critical behaviour with respect to the RSB effects.

We consider an $O(p)$-symmetric Ginzburg-LandauWilson model of a spin system with weak quenched disorder near critical point given by the Hamiltonian

$$
\begin{aligned}
H & =\int d^{d} x\left\{\frac{1}{2} \sum_{i=1}^{p}\left[\nabla \phi_{i}(x)\right]^{2}+\frac{1}{2}[\tau-\delta \tau(x)] \sum_{i=1}^{p} \phi_{i}^{2}(x)\right. \\
& \left.+\frac{1}{4} g \sum_{i, j=1}^{p} \phi_{i}^{2}(x) \phi_{j}^{2}(x)\right\},
\end{aligned}
$$


where $\phi_{i}(x)$ is the $p$-component order parameter and $\delta \tau(x)$ is the Gaussian-distributed random transition temperature with the second moment of distribution $\left\langle\left\langle(\delta \tau(x))^{2}\right\rangle\right\rangle \sim u$ defined by the positive constant $u$ which is proportional to the concentration of defects. The use of the standard replica trick gives us the possibility to easily average over the disorder and reduce the task of statistical description of the weakly disordered system with the Hamiltonian (1) to the homogeneous system with the effective Hamiltonian

$$
\begin{aligned}
H_{n} & =\int d^{d} x\left\{\frac{1}{2} \sum_{i=1}^{p} \sum_{a=1}^{n}\left[\left[\nabla \phi_{i}^{a}(x)\right]^{2}+\tau\left[\phi_{i}^{a}(x)\right]^{2}\right]\right. \\
& \left.+\frac{1}{4} \sum_{i, j=1}^{p} \sum_{a, b=1}^{n} g_{a b}\left[\phi_{i}^{a}(x)\right]^{2}\left[\phi_{j}^{b}(x)\right]^{2}\right\}
\end{aligned}
$$

which is a functional of $n$ replications of the original order parameter with an additional vertex $u$ in the RS matrix $g_{a b}=g \delta_{a b}-u$. The properties of the original disordered system are obtained in the replica number limit $n \rightarrow 0$. The following standard RG procedure based on the statistical calculation of the contribution to the partition function of long-wavelength order parameter fluctuations around the global minimum state with $\phi(x)=0$ gives us the possibility to find the various types of critical behaviour and conditions of their stability and carry out the calculation of critical exponents.

However, as shown in [6-8], the fluctuations of random transition temperature $\delta \tau(x)$ for $[\tau-\delta \tau(x)]<0$ can lead to realization in a system of numerous regions with $\phi(x) \neq 0$ displayed through the numerous local minimalenergy configurations separated from the ground state by finite barriers. In this case the direct application of the traditional RS RG scheme may be questioned. For statistical description of such systems near the phase transition point the Parisi RSB scheme was used in [6-8] by analogy with spin glasses [11]. It was argued that spontaneous RSB can occur due to the interaction of the fluctuating fields with the local non-perturbative degrees of freedom from the multiple-local-minimum solutions of the mean-field equations. It was shown that the summation over these solutions in the replica partition function can provide the additional non-trivial RSB potential $\sum_{a, b} g_{a b} \phi_{a}^{2} \phi_{b}^{2}$ in which the matrix $g_{a b}$ has the Parisi RSB structure [11]. According to the technique of the Parisi RSB algebra, in the limit $n \rightarrow 0$ the matrix $g_{a b}$ is parametrized in terms of its diagonal elements $\tilde{g}$ and the off-diagonal function $g(x)$ defined in the interval $0<x<1: g_{a b} \rightarrow(\tilde{g}, g(x))$. The operations with the matrices $g_{a b}$ are given by the following rules:

$$
\begin{aligned}
& g_{a b}^{k} \rightarrow\left(\tilde{g}^{k} ; g^{k}(x)\right), \quad\left(\hat{g}^{2}\right)_{a b}=\sum_{c=1}^{n} g_{a c} g_{c b} \rightarrow(\tilde{c} ; c(x)) \\
& \left(\hat{g}^{3}\right)_{a b}=\sum_{c, d=1}^{n} g_{a c} g_{c d} g_{d b} \rightarrow(\tilde{d} ; d(x))
\end{aligned}
$$

where

$$
\begin{aligned}
\tilde{c} & =\tilde{g}^{2}-\int_{0}^{1} d x g^{2}(x), \\
c(x) & =2\left[\tilde{g}-\int_{0}^{1} d y g(y)\right] g(x)-\int_{0}^{x} d y[g(x)-g(y)]^{2}, \\
\tilde{d} & =\tilde{c} \tilde{g}-\int_{0}^{1} d x c(x) g(x), \\
d(x) & =\left[\tilde{g}-\int_{0}^{1} d y g(y)\right] c(x)+\left[\tilde{c}-\int_{0}^{1} d y c(y)\right] g(x) \\
& -\int_{0}^{x} d y[g(x)-g(y)][c(x)-c(y)] .
\end{aligned}
$$

The RS situation corresponds to the case $g(x)=$ const independent of $x$.

We carried out the field-theoretical RG description of the $d$-dimensional model with the effective replicated Hamiltonian (2) in which the matrix $g_{a b}$ has the RSB structure in the two-loop approximation. We considered the most important cases with $d=3$ and $d=2$. The case of systems with the arbitrary dimension $3<d<4$ was considered also for the clearing up of the range where the $\varepsilon$-expansion results could be applicable. In the fieldtheoretic approach the asymptotic critical behavior of systems in the fluctuation region are determined by the Callan-Symanzik RG equation for the vertex parts of the irreducible Green's functions. To calculate the $\beta$ functions as functions of the renormalized elements of the matrix $g_{a b}$ appearing in the RG equation, we used the method based on the Feynman diagram technique and the renormalization procedure [12]. However, the renormalization procedure for vertex functions is made difficult because of complicated expressions (3)-(4) for the operations with the matrices $g_{a b}$. The steplike structure of the function $g(x)$ revealed in [6-8] gives us the possibility to realize the renormalization procedure. In this paper we considered only the matrices $g_{a b}$ which have the structure known as the one-step RSB with the function $g(x)$ which looks as:

$$
g(x)= \begin{cases}g_{0}, & 0 \leq x<x_{0} \\ g_{1}, & x_{0}<x \leq 1\end{cases}
$$

where $0 \leq x_{0} \leq 1$ is the coordinate of the step and it remains an arbitrary parameter. The value of $x_{0}$ is not changed during the renormalization procedure and remains the same as in the starting function $g_{0}(x)$. In consequence the RG transformations of the effective replicated Hamiltonian with RSB potentials are determined by the three parameters $\tilde{g}, g_{0}$ and $g_{1}$.

The critical properties of the model can be extracted from the coefficients $\beta_{i}\left(\tilde{g}, g_{0}, g_{1}\right)(i=1,2,3)$, 
$\gamma_{\phi}\left(\tilde{g}, g_{0}, g_{1}\right)$, and $\gamma_{\phi^{2}}\left(\tilde{g}, g_{0}, g_{1}\right)$ of the Callan-Symanzik $\mathrm{RG}$ equation. We obtained the $\beta$ and $\gamma$ functions in the two-loop approximation in the form of the expansion series in renormalized parameters $\tilde{g}, g_{0}$ and $g_{1}$

$$
\begin{aligned}
& \beta_{1}=-\tilde{g}+(8+p) \tilde{g}^{2}-p x_{0} g_{0}{ }^{2}-p\left(1-x_{0}\right) g_{1}{ }^{2}+[(8 f-40 h+20) p \\
& +16 f-176 h+88] \tilde{g}^{3}+(24 h-8 f-12) x_{0} p \tilde{g} g_{0}{ }^{2}+(24 h-8 f \\
& -12)\left(1-x_{0}\right) p \tilde{g} g_{1}{ }^{2}-(16 h-8) x_{0} p g_{0}{ }^{3}-(16 h-8)\left(1-x_{0}\right) p g_{1}{ }^{3} \text {, } \\
& \beta_{2}=-g_{0}+(4+2 p) \tilde{g} g_{0}+\left(2 p x_{0}-4\right) g_{0}{ }^{2}+2\left(1-x_{0}\right) p g_{0} g_{1} \\
& +[(8 f-48 h+28) p+16 f-48 h+24] \tilde{g}^{2} g_{0}-\left[\left((32 h-16) x_{0}\right.\right. \\
& +8-32 h) p+48-96 h] \tilde{g} g_{0}{ }^{2}-(32 h-16)\left(1-x_{0}\right) p \tilde{g} g_{0} g_{1} \\
& +\left[(48 h-8 f-20) x_{0} p-32 h+16\right] g_{0}{ }^{3}+(32 h-8)\left(1-x_{0}\right) p g_{0}{ }^{2} g_{1} \\
& +(16 h-12-8 f)\left(1-x_{0}\right) p g_{0} g_{1}^{2}, \\
& \beta_{3}=-g_{1}+p x_{0} g_{0}{ }^{2}-\left[p\left(x_{0}-2\right)+4\right] g_{1}{ }^{2}+(4+2 p) \tilde{g} g_{1}+[(8 f-48 h \\
& +28) p+16 f-48 h+24] g_{1} \tilde{g}^{2}-(16 h-8) x_{0} p \tilde{g} g_{0}{ }^{2}-\left[\left((8-16 h) x_{0}\right.\right. \\
& -8) p+48-96 h] u_{0} g_{1}{ }^{2}+(16 h-8) x_{0} p g_{0}{ }^{3}+(8 h-8 f-4) x_{0} p g_{1} g_{0}{ }^{2} \\
& +\left[(8 f-24 h+12) x_{0} p+(48 h-8 f-20) p+16-32 h\right] g_{1}{ }^{3}, \\
& \gamma_{\phi}=4(4-d) f(d)\left[(p+2) \tilde{g}^{2}-p x_{0} g_{0}{ }^{2}-p\left(1-x_{0}\right) g_{1}{ }^{2}\right] \text {, } \\
& \gamma_{\phi^{2}}=(4-d)\left[(p+2) \tilde{g}+p x_{0} g_{0}+p\left(1-x_{0}\right) g_{1}-2(6 h-2 f-3)\left((p+2) \tilde{g}^{2}\right.\right. \\
& \left.\left.-p x_{0} g_{0}^{2}-p\left(1-x_{0}\right) g_{1}^{2}\right)\right]
\end{aligned}
$$

where

$$
\begin{aligned}
& f(d)=-\left.\frac{1}{J^{2}} \frac{\partial}{\partial k^{2}} \int \frac{d^{d} k_{1} d^{d} k_{2}}{\left(k_{1}^{2}+1\right)\left(k_{2}^{2}+1\right)\left(\left(k_{1}+k_{2}+k\right)^{2}+1\right)}\right|_{k^{2}=0}, \\
& h(d)=\frac{1}{J^{2}} \int \frac{d^{d} k_{1} d^{d} k_{2}}{\left(k_{1}^{2}+1\right)^{2}\left(k_{2}^{2}+1\right)\left(\left(k_{1}+k_{2}\right)^{2}+1\right)}, \quad J=\int d^{d} k /\left(k^{2}+1\right)^{2}, \\
& f(d=3)=\frac{2}{27}, \quad h(d=3)=\frac{2}{3}, \quad f(d=2)=0.11464, \quad h(d=2)=0.78130 \quad[13]
\end{aligned}
$$

and in (6) was made the redefinition $g_{a b} \rightarrow g_{a b} / J$. By analogy with papers [6-8] we changed $g_{a \neq b} \rightarrow-g_{a \neq b}$ in expressions (6) for the $\beta$ and $\gamma$ functions, so that the off-diagonal elements $g_{a \neq b}$ would be positively defined. Also, we numerically calculated the integrals in the expressions for $f(d)$ and $h(d)$ for $3 \leq d<4$. The numerical values of 2-loop integrals for the case of non-integer space dimensionalities $d$ are given also in [14].
It is well known that perturbation series are asymptotic series, and that the vertices describing the interaction of the order parameter fluctuations in the fluctuating region $\tau \rightarrow 0$ are large enough that expressions (6) cannot be used directly. For this reason, to extract the required physical information from the obtained expressions, we employed the Padé-Borel approximation of the summation of asymptotic series extended to the 
multiparameter case. The direct and inverse Borel transformations for the multiparameter case have the form

$$
\begin{aligned}
& f\left(\tilde{g}, g_{0}, g_{1}\right)=\sum_{i, j, k} c_{i j k} \tilde{g}^{i} g_{0}^{j} g_{1}^{k}=\int_{0}^{\infty} e^{-t} F\left(\tilde{g} t, g_{0} t, g_{1} t\right) d t \\
& F\left(\tilde{g}, g_{0}, g_{1}\right)=\sum_{i, j, k} \frac{c_{i j k}}{(i+j+k) !} \tilde{g}^{i} g_{0}^{j} g_{1}^{k}
\end{aligned}
$$

A series in the auxiliary variable $\theta$ is introduced for analytical continuation of the Borel transform of the function:

$$
\tilde{F}\left(\tilde{g}, g_{0}, g_{1}, \theta\right)=\sum_{k=0}^{\infty} \theta^{k} \sum_{i=0}^{k} \sum_{j=0}^{k-i} \frac{c_{i, j, k-i-j}}{k !} \tilde{g}^{i} g_{0}^{j} g_{1}^{k-i-j}
$$

to which the $[\mathrm{L} / \mathrm{M}]$ Padé approximation is applied at the point $\theta=1$. To perform the analytical continuation, the Padé approximant of [L/1] type may be used which is known to provide rather good results for various Landau-Wilson models (see, e.g., [15,16]). The property of preserving the symmetry of a system during application of the Padé approximation by the $\theta$ method, as in [15], has become important for multivertex models. We used the $[2 / 1]$ approximant to calculate the $\beta$ functions in the two-loop approximation.

The nature of the critical behaviour is determined by the existence of a stable FP satisfying the system of equations

$$
\beta_{i}\left(\tilde{g}^{*}, g_{0}^{*}, g_{1}^{*}\right)=0 \quad(i=1,2,3) .
$$

We have found three types of non-trivial FP in the physical region of parameter space $\tilde{g}^{*}, g_{0}^{*}, g_{1}^{*} \geq 0$ for different values of $p=1,2,3$, which are presented in Tables 1-4 (the exception was made in the case with $p=3$ for $d=3.0$, when presented in Table 3 the coordinates of type II and type III FPs are characterized by unphysical negative values of $g_{0}^{*}$ and $g_{1}^{*}$ ). Type I with $\tilde{g}^{*} \neq 0, g_{0}^{*}=g_{1}^{*}=0$ corresponds to the RS FP of a pure system, type II with $\tilde{g}^{*} \neq 0, g_{0}^{*}=g_{1}^{*} \neq 0$ is a disorderinduced RS FP and type III with $\tilde{g}^{*} \neq 0, g_{0}^{*}=0, g_{1}^{*} \neq 0$ corresponds to the one-step RSB FP. The values of parameters $\tilde{g}^{*}, g_{1}^{*}$ for the one-step RSB FP depend on the coordinate of the step $x_{0}$, and we present in Tables $1-$ 4 the received values of these parameters in the range $0 \leq x_{0} \leq 1$ with changes through the step $\Delta x_{0}=0,1$.

The type of critical behaviour of this disordered system for each value of $p$ is determined by the stability of the corresponding FP. The requirement that the FP be stable reduces to the condition that the real part of the eigenvalues $\lambda_{i}$ of the matrix

$$
B_{i, j}=\frac{\partial \beta_{i}\left(\tilde{g}^{*}, g_{0}^{*}, g_{1}^{*}\right)}{\partial g_{j}}
$$

must be positive.

Analysis of the values $\lambda_{i}$ for FPs presented in Tables 1-4 shows that for $3 \mathrm{D}$ and $2 \mathrm{D}$ Ising models $(p=1)$ the disorder-induced RS FPs are stable [17]. However, this type FP will lose stability for Ising model with the dimension greater than the marginal dimension $d_{c}=3.986$. As for other types of FPs are not stable in the range with dimension $d$ from 3 to 4 , therefore the critical behaviour of weakly disordered Ising systems will be unstable with respect to the RSB effects when dimension $d$ takes on the value greater than 3.986 .

\begin{tabular}{|c|c|c|c|c|cc|c|}
\hline Type & $x_{0}$ & $\tilde{g}^{*}$ & $g_{0}^{*}$ & $g_{1}^{*}$ & $\lambda_{1}$ & $\lambda_{2}$ & $\lambda_{3}$ \\
\hline \hline I & & 0.1774 & 0 & 0 & 0.6536 & -0.1692 & -0.1692 \\
\hline II & & 0.1844 & 0.0812 & 0.0812 & $0.5253 \pm 0.0893 i$ & 0.2112 \\
\hline III & 0.0 & 0.1844 & 0 & 0.0812 & $0.5253 \pm 0.0893 i$ & -0.0392 \\
& 0.1 & 0.1840 & 0 & 0.0829 & $0.5352 \pm 0.0983 i$ & -0.0492 \\
& 0.2 & 0.1835 & 0 & 0.0846 & $0.5471 \pm 0.1067 i$ & -0.0599 \\
& 0.3 & 0.1830 & 0 & 0.0863 & $0.5607 \pm 0.1133 i$ & -0.0712 \\
& 0.4 & 0.1824 & 0 & 0.0880 & $0.5765 \pm 0.1180 i$ & -0.0832 \\
& 0.5 & 0.1817 & 0 & 0.0895 & $0.5951 \pm 0.1203 i$ & -0.0959 \\
& 0.6 & 0.1810 & 0 & 0.0910 & $0.6172 \pm 0.1189 i$ & -0.1093 \\
& 0.7 & 0.1802 & 0 & 0.0924 & $0.6439 \pm 0.1114 i$ & -0.1234 \\
& 0.8 & 0.1793 & 0 & 0.0936 & $0.6760 \pm 0.0921 i$ & -0.1381 \\
& 0.9 & 0.1784 & 0 & 0.0947 & $0.7135 \pm 0.0353 i$ & -0.1534 \\
& 1.0 & 0.1774 & 0 & 0.0957 & 0.8573 & 0.6536 & -0.1692 \\
\hline
\end{tabular}

a) dimension $d=3.0$

\begin{tabular}{|c|c|c|c|c|cc|c|}
\hline Type & $x_{0}$ & $\tilde{g}^{*}$ & $g_{0}^{*}$ & $g_{1}^{*}$ & $\lambda_{1}$ & $\lambda_{2}$ & $\lambda_{3}$ \\
\hline \hline I & & 0.0917 & 0 & 0 & 0.6315 & -0.4163 & -0.4163 \\
\hline II & & 0.1231 & 0.1090 & 0.1090 & $0.6986 \pm 0.1311 i$ & 0.0022 \\
\hline III & 0.0 & 0.1231 & 0 & 0.1090 & $0.7047 \pm 0.1069 i$ & -0.0363 \\
\hline
\end{tabular}

b) dimension $d=3.985$ 


\begin{tabular}{|c|c|c|c|c|cc|c|}
\hline Type & $x_{0}$ & $\tilde{g}^{*}$ & $g_{0}^{*}$ & $g_{1}^{*}$ & $\lambda_{1}$ & $\lambda_{2}$ & $\lambda_{3}$ \\
\hline \hline I & & 0.0916 & 0 & 0 & 0.6318 & -0.4165 & -0.4165 \\
\hline II & & 0.1230 & 0.1092 & 0.1092 & $0.6895 \pm 0.1453 i$ & -0.0076 \\
\hline III & 0.0 & 0.1230 & 0 & 0.1092 & $0.7018 \pm 0.0935 i$ & -0.0359 \\
\hline
\end{tabular}

c) dimension $d=3.986$

Table 1. Coordinates of the FPs and eigenvalues of the stability matrix for $p=1$.

\begin{tabular}{|c|c|c|c|c|c|c|c|}
\hline Type & $x_{0}$ & $\tilde{g}^{*}$ & $g_{0}^{*}$ & $g_{1}^{*}$ & $\lambda_{1}$ & $\lambda_{2}$ & $\lambda_{3}$ \\
\hline \hline I & & 0.155830 & 0 & 0 & 0.667315 & -0.001672 & -0.001672 \\
\hline II & & 0.155831 & 0.000584 & 0.000584 & 0.667312 & 0.001682 & 0.000004 \\
\hline III & 0.0 & 0.155831 & 0 & 0.000584 & 0.667313 & 0.001683 & -0.000001 \\
& 0.1 & 0.155831 & 0 & 0.000614 & 0.667313 & 0.001684 & -0.000088 \\
& 0.2 & 0.155831 & 0 & 0.000648 & 0.667313 & 0.001685 & -0.000186 \\
& 0.3 & 0.155831 & 0 & 0.000686 & 0.667313 & 0.001686 & -0.000296 \\
& 0.4 & 0.155831 & 0 & 0.000729 & 0.667313 & 0.001687 & -0.000419 \\
& 0.5 & 0.155831 & 0 & 0.000778 & 0.667313 & 0.001687 & -0.000559 \\
& 0.6 & 0.155831 & 0 & 0.000833 & 0.667313 & 0.001688 & -0.000717 \\
& 0.7 & 0.155831 & 0 & 0.000896 & 0.667314 & 0.001690 & -0.000901 \\
& 0.8 & 0.155831 & 0 & 0.000971 & 0.667314 & 0.001692 & -0.001116 \\
& 0.9 & 0.155831 & 0 & 0.001058 & 0.667315 & 0.001694 & -0.001369 \\
& 1.0 & 0.155830 & 0 & 0.001163 & 0.667316 & 0.001696 & -0.001672 \\
\hline
\end{tabular}

a) dimension $d=3.0$

\begin{tabular}{|c|c|c|c|c|c|c|c|}
\hline Type & $x_{0}$ & $\tilde{g}^{*}$ & $g_{0}^{*}$ & $g_{1}^{*}$ & $\lambda_{1}$ & $\lambda_{2}$ & $\lambda_{3}$ \\
\hline \hline I & & 0.1499955 & 0 & 0 & 0.689608 & -0.009539 & -0.009539 \\
\hline II & & 0.1500170 & 0.00325 & 0.00325 & 0.689535 & 0.009887 & -0.000003 \\
\hline III & 0.0 & 0.1500170 & 0 & 0.00325 & 0.689535 & 0.009887 & 0.000109 \\
& 0.1 & 0.1500169 & 0 & 0.00341 & 0.689535 & 0.009899 & -0.000401 \\
& 0.2 & 0.1500167 & 0 & 0.00360 & 0.689536 & 0.009926 & -0.000961 \\
\hline
\end{tabular}

b) dimension $d=3.10$

\begin{tabular}{|c|c|c|c|c|c|c|c|}
\hline Type & $x_{0}$ & $\tilde{g}^{*}$ & $g_{0}^{*}$ & $g_{1}^{*}$ & $\lambda_{1}$ & $\lambda_{2}$ & $\lambda_{3}$ \\
\hline \hline I & & 0.089762 & 0 & 0 & 1.119442 & -0.133591 & -0.133591 \\
\hline II & & 0.092307 & 0.036991 & 0.036991 & 1.103421 & 0.227335 & -0.025378 \\
\hline III & 0.0 & 0.092307 & 0 & 0.036991 & 1.103421 & 0.227335 & 0.030783 \\
& 0.1 & 0.092270 & 0 & 0.038723 & 1.102142 & 0.235506 & 0.021563 \\
& 0.2 & 0.092205 & 0 & 0.040559 & 1.100913 & 0.244667 & 0.011135 \\
& 0.3 & 0.092108 & 0 & 0.042500 & 1.099845 & 0.254810 & -0.000648 \\
& 0.4 & 0.091970 & 0 & 0.044547 & 1.099106 & 0.265820 & -0.013939 \\
\hline
\end{tabular}

c) dimension $d=3.999$

Table 2. Coordinates of the FPs and eigenvalues of the stability matrix for $p=2$.

For the 3D $X Y$ model $(p=2)$ the obtained small positive eigenvalues $\lambda_{i}$ show that the disorder-induced RS FP is characterized by weak stability. However, when the dimension $d$ of the system is greater than the marginal dimension $d_{c}=3.1$ the RSB FP is stable. In this case, the critical behaviour of weakly disordered systems will be non-universal and dependent on the coordinate of the step $x_{0}$ and, as consequence, on impurity concentration.
Analysis of FP stability shows that RSB FP (type III) is stable only in the range of changes $x_{0}$ from 0 to $x_{c}$, where $x_{c}$ is a marginal value depending on dimension system. So, for the weakly disordered system dimension of $d=3.1, x_{c}$ is equal 0.1 , and for $d=3.999 x_{c}=0.3$. In the range of $x_{0}$ from $x_{c}$ to 1 all types of FPs lose their stability. However, we must notice that the results, which we received for the disordered $X Y$ model, will be 
corrected in the higher field-theory orders of approximation. We believe that the critical behaviour of the $3 \mathrm{D}$ XY model will be determined by the RS FP of a pure system (I), but not by the disorder-induced RS FP (II), obtained in the two-loop order. Two facts indicate this: the weak stability of the disorder-induced RS FP and that in the two-loop approximation the marginal value of $p_{c}=2.0114$ for the borderline between regions of stability for the disorder-induced RS FP and the RS FP of a pure system. In the higher orders of approximation the marginal value of $p_{c}<2$, such as the specific heat exponent $\alpha>0$ for the pure $3 \mathrm{D} X Y$ model. Also, our results about influence of the RSB effects for XY model with $d>3$ will be corrected in the higher orders of approximation, but we think these changes will be quantitative only.

For the 3D Heisenberg model $(p=3)$ the RS FP of a pure system is stable and both other types of FP are characterized by unphysical negative values of coordinates $g_{0}^{*}$ and $g_{1}^{*}$. Only for the dimension $d_{c}=3.999$ the values of coordinates $g_{0}^{*}$ and $g_{1}^{*}$ become positive and simultaneously RSB FP type III become stable in the range of changes $x_{0}$ from 0 to 0.4 . In the range of $x_{0}$ from 0.4 to 1 there exist no stable FPs.

\begin{tabular}{|c|c|c|c|c|c|c|c|}
\hline Type & $x_{0}$ & $\tilde{g}^{*}$ & $g_{0}^{*}$ & $g_{1}^{*}$ & $\lambda_{1}$ & $\lambda_{2}$ & $\lambda_{3}$ \\
\hline \hline I & & 0.1383 & 0 & 0 & 0.6814 & 0.1315 & 0.1315 \\
\hline II & & 0.1419 & -0.0359 & -0.0359 & 0.6727 & -0.0891 & -0.1450 \\
\hline III & 0.0 & 0.1419 & 0 & -0.0359 & 0.6727 & -0.0891 & -0.0058 \\
& 0.1 & 0.1420 & 0 & -0.0382 & 0.6727 & -0.0865 & 0.0011 \\
& 0.2 & 0.1420 & 0 & -0.0408 & 0.6728 & -0.0836 & 0.0088 \\
& 0.3 & 0.1421 & 0 & -0.0439 & 0.6730 & -0.0802 & 0.0175 \\
& 0.4 & 0.1420 & 0 & -0.0474 & 0.6734 & -0.0764 & 0.0273 \\
& 0.5 & 0.1420 & 0 & -0.0516 & 0.6738 & -0.0719 & 0.0385 \\
& 0.6 & 0.1418 & 0 & -0.0565 & 0.6745 & -0.0668 & 0.0515 \\
& 0.7 & 0.1415 & 0 & -0.0625 & 0.6755 & -0.0606 & 0.0667 \\
& 0.8 & 0.1409 & 0 & -0.0699 & 0.6768 & -0.0533 & 0.0845 \\
& 0.9 & 0.1400 & 0 & -0.0793 & 0.6787 & -0.0443 & 0.1058 \\
& 1.0 & 0.1383 & 0 & -0.0915 & 0.6814 & -0.0331 & 0.1315 \\
\hline
\end{tabular}

a) dimension $d=3.0$

\begin{tabular}{|c|c|c|c|c|c|c|c|}
\hline Type & $x_{0}$ & $\tilde{g}^{*}$ & $g_{0}^{*}$ & $g_{1}^{*}$ & $\lambda_{1}$ & $\lambda_{2}$ & $\lambda_{3}$ \\
\hline \hline I & & 0.081989 & 0 & 0 & 1.113633 & -0.000820 & -0.000820 \\
\hline II & & 0.081989 & 0.000171 & 0.000171 & 1.113633 & 0.000822 & -0.000228 \\
\hline III & 0.0 & 0.081989 & 0 & 0.000171 & 1.113633 & 0.000822 & 0.000228 \\
& 0.1 & 0.081989 & 0 & 0.000183 & 1.113633 & 0.000822 & 0.000188 \\
& 0.2 & 0.081989 & 0 & 0.000196 & 1.113633 & 0.000823 & 0.000142 \\
& 0.3 & 0.081989 & 0 & 0.000212 & 1.113633 & 0.000823 & 0.000088 \\
& 0.4 & 0.081989 & 0 & 0.000230 & 1.113633 & 0.000823 & 0.000025 \\
& 0.5 & 0.081989 & 0 & 0.000251 & 1.113633 & 0.000823 & -0.000050 \\
& 0.6 & 0.081989 & 0 & 0.000277 & 1.113633 & 0.000824 & -0.000140 \\
& 0.7 & 0.081989 & 0 & 0.000309 & 1.113633 & 0.000824 & -0.000251 \\
& 0.8 & 0.081989 & 0 & 0.000350 & 1.113633 & 0.000825 & -0.000391 \\
& 0.9 & 0.081989 & 0 & 0.000402 & 1.113633 & 0.000826 & -0.000574 \\
& 1.0 & 0.081989 & 0 & 0.000473 & 1.113633 & 0.000828 & -0.000820 \\
\hline
\end{tabular}

b) dimension $d=3.999$

Table 3. Coordinates of the FPs and eigenvalues of the stability matrix for $p=3$.

Thus, the investigations carried out in the two-loop approximation show the stability of the critical behaviour of two- and three-dimensional weakly disordered systems with respect to the $\mathrm{RSB}$ effects. In dilute Ising-like systems the disorder-induced critical behaviour is realized with RS FP. The weak disorder is irrelevant for the criti- cal behaviour of systems with multicomponent order parameter although the proof for $3 \mathrm{D} X Y$-like systems demands calculations in the higher orders of approximation. Our conclusions coincide with results of paper [9] for the $2 \mathrm{D}$ random Ising model, but contradict with results of papers [6-8] for $3 \mathrm{D}$ disordered systems. 


\begin{tabular}{|c|c|c|c|c|c|c|c|}
\hline Type & $x_{0}$ & $\tilde{g}^{*}$ & $g_{0}^{*}$ & $g_{1}^{*}$ & $\lambda_{1}$ & $\lambda_{2}$ & $\lambda_{3}$ \\
\hline \hline I & & 0.2694 & 0 & 0 & 0.4612 & -0.0952 & -0.0952 \\
\hline II & & 0.2736 & -0.0646 & -0.0646 & 0.4463 & 0.1621 & 0.0570 \\
\hline III & 0.0 & 0.2736 & 0 & -0.0646 & 0.4463 & 0.1621 & -0.0251 \\
& 0.1 & 0.2734 & 0 & -0.0665 & 0.4466 & 0.1653 & -0.0300 \\
& 0.2 & 0.2732 & 0 & -0.0685 & 0.4470 & 0.1688 & -0.0352 \\
& 0.3 & 0.2729 & 0 & -0.0706 & 0.4476 & 0.1726 & -0.0409 \\
& 0.4 & 0.2726 & 0 & -0.0728 & 0.4484 & 0.1768 & -0.0470 \\
& 0.5 & 0.2723 & 0 & -0.0751 & 0.4494 & 0.1815 & -0.0535 \\
& 0.6 & 0.2719 & 0 & -0.0775 & 0.4507 & 0.1866 & -0.0606 \\
& 0.7 & 0.2714 & 0 & -0.0801 & 0.4524 & 0.1922 & -0.0682 \\
& 0.8 & 0.2708 & 0 & -0.0827 & 0.4546 & 0.1984 & -0.0765 \\
& 0.9 & 0.2701 & 0 & -0.0854 & 0.4575 & 0.2053 & -0.0855 \\
& 1.0 & 0.2694 & 0 & -0.0882 & 0.4612 & 0.2129 & -0.0952 \\
\hline
\end{tabular}

Table 4. Coordinates of the FPs and eigenvalues of the stability matrix for the 2D Ising model.

The influence of the RSB effects are realized only for disordered systems with the dimension $d$ larger than 3 . In this case the values of the marginal dimension $d_{c}$ depend on the coordinate of the step $x_{0}$ and the number of order parameter components $p$. For $d$ greater than marginal dimension $d_{c}$ our results qualitatively correspond to results of papers [6-8] received on the basis of the $\varepsilon$-expansion. So, for Ising systems the effects of RSB destroy a stable critical behaviour. For $X Y$ and Heisenberg models the range of non-universal critical behaviour exists for the coordinate of the step $x_{0}$ from 0 to threshold value $x_{c}$ depending on dimension $d$. The stable critical behaviour is not realized for the coordinate of the step $x_{0}$ out of this range just as for Ising systems.
We assume that with the increasing of defect concentration the marginal dimension $d_{c}$ could be reduced until the value $d_{c}=3$ for some threshold concentration of defects. In this case the effects of RSB will be essential.

\section{ACKNOWLEDGMENT}

This work was supported in part by the Russian Foundation for Basic Research through Grants No. 00-0216455 (V.V.P.) and No. 01-02-06206 (P.V.P.) and the Ministry of Education of the Russian Federation through Grant No. E00-3.2-43.
[1] A. B. Harris, J. Phys. C 7, 1671 (1974).

[2] A. B. Harris, T. C. Lubensky, Phys. Rev. Lett. 33, 1540 (1974).

[3] D. E. Khmelnitskii, Sov. Phys. JETP 41981 (1975).

[4] J. Emery, Phys. Rev. B 11, 239 (1975).

[5] G. Grinstein, A. Luther, Phys. Rev. B 13, 1329 (1976).

[6] Vik. S. Dotsenko, A. B. Harris, D. Sherrington, R. B. Stinchcombe, J. Phys. A 28, 3093 (1995).

[7] Vik. S. Dotsenko, D. E. Feldman, J. Phys. A 28, 5183 (1995).

[8] Vik. S. Dotsenko, Usp. Fiz. Nauk 165, 481 (1995).

[9] D. E. Feldman, A. V. Izyumov, Vik. S. Dotsenko, preprint cond-mat/9512158.

[10] V. V. Prudnikov, A. V. Ivanov, A. A. Fedorenko, Sov. Phys. JETP Lett. 66835 (1997); V. V. Prudnikov, S. V. Belim, A. V. Ivanov, E. V. Osintsev, A. A. Fedorenko, Sov. Phys. JETP 87527 (1998); V. V. Prudnikov, P. V. Prudnikov, A. A. Fedorenko, Sov. Phys. JETP Lett. 68950 (1998); Phys. Rev. B 62, 8777 (2000).

[11] M. Mezard, G. Parisi, M. Virasoro, Spin-Glass Theory and Beyond (Singapore, World Scientific, 1987);
M. Mezard, G. Parisi, J.Phys. I (France) 1, 809 (1991); M. Mezard, A. P. Young, Europhys. Lett. 18, 653 (1992); Vik. S. Dotsenko, Usp. Fiz. Nauk Sov. Phys. Usp. 163, 1 (1993): Vik. S. Dotsenko, Introduction to the Theory of Spin-Glasses and Neural Networks (Singapore, World Scientific, 1994).

[12] J. Zinn-Justin, Quantum field theory and critical phenomena (Clarendon Press, Oxford, 1996).

[13] B. G. Nickel, D. I. Meiron, G. A. Baker, Compilation of 2pt and 4pt graphs for continuous spin model (University of Guelph Report, 1977).

[14] Yu. Holovatch, M. Shpot, J. Stat. Phys 66, 847 (1992).

[15] S. A. Antonenko, A. I. Sokolov, Phys. Rev. B 49, 15901 (1994); A. I. Sokolov, K. B. Varnashev, A. I. Mudrov. Int. J. Mod. Phys. B 12, 1365 (1998); A. I. Sokolov, K. B. Varnashev, Phys. Rev. B 59. 8363 (1999).

[16] G. A. Baker, B. G. Nickel, D. I. Meiron, Phys. Rev. B 17, 1365 (1978).

[17] V. V. Prudnikov, P. V. Prudnikov, A. A. Fedorenko, Phys. Rev. B 63, 184201 (2001). 


\title{
КРИТИЧНА ПОВЕДІНКА СЛАБОНЕВПОРЯДКОВАНИХ СИСТЕМ ІЗ ПОТЕНЩЯЛАМИ З ПОРУШЕНОЮ РЕПЛІЧНОЮ СИМЕТРІЕЮ
}

\author{
П. В. Прудніков, В. В. Прудніков \\ Кафедра теоретичной фізики, Омсъкий державний університет \\ Пр. Миру, 55a, Омсък, 644077, Росія
}

Подано теоретико-польовий опис критичної поведінки слабоневпорядкованих систем. А саме, для трита двовимірних систем у двопетлевому наближенні проведено ренормалізапійний аналіз ефективного гамільтоніяна моделі з потенціялами з порушеною реплічною симетрією. Для односходинкового порушення реплічної симетрії за, допомогою пересумовування Паде-Бореля виявлено нерухомі точки, що відповідають стійкості різних типів критичної поведінки. Аналіз нерухомих точок показав стійкість критичної поведінки слабоневпорядкованих три- та двовимірних систем щодо ефектів порушення реплічної симетрії та реалізащію старого сценарію впливу безладу на критичну поведінку. Для систем із довільною вимірністю від 3 до 4 та для кожного значення $p$-компонентного параметра впорядування без застосування $\varepsilon$-розкладу знайдено інтервали критичної поведінки, які визначаються ефектами порушення реплічної симетрії. Проведено порівняння з результатами отриманими на основі $\varepsilon$-розкладу. 